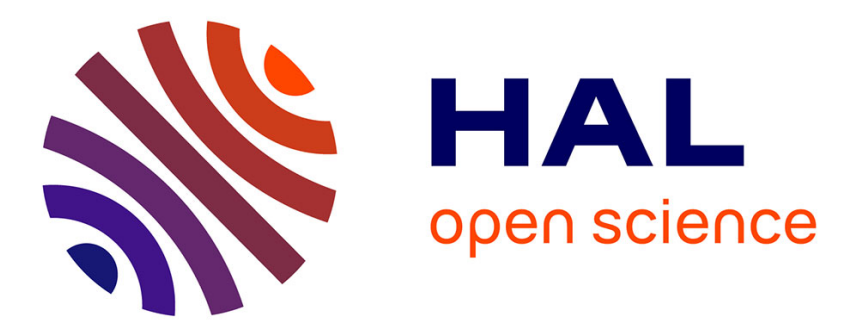

\title{
2D interaction of a gaussian beam with a dichroic surface for the modeling of quasi-optical systems
}

Kevin Elis, Alexandre Chabory, Jérôme Sokoloff, Sylvain Bolioli

\section{To cite this version:}

Kevin Elis, Alexandre Chabory, Jérôme Sokoloff, Sylvain Bolioli. 2D interaction of a gaussian beam with a dichroic surface for the modeling of quasi-optical systems. ICEAA 2011, 13th International Conference on Electromagnetics in Advanced Applications, Sep 2011, Torino, Italy. pp 682-685, 10.1109/ICEAA.2011.6046425 . hal-01022222

\section{HAL Id: hal-01022222 \\ https://hal-enac.archives-ouvertes.fr/hal-01022222}

Submitted on 22 Sep 2014

HAL is a multi-disciplinary open access archive for the deposit and dissemination of scientific research documents, whether they are published or not. The documents may come from teaching and research institutions in France or abroad, or from public or private research centers.
L'archive ouverte pluridisciplinaire HAL, est destinée au dépôt et à la diffusion de documents scientifiques de niveau recherche, publiés ou non, émanant des établissements d'enseignement et de recherche français ou étrangers, des laboratoires publics ou privés. 


\title{
2D interaction of a Gaussian Beam with a dichroic surface for the modeling of quasi-optical systems
}

\author{
K. Elis ${ }^{* \dagger \ddagger}$, A. Chabory ${ }^{\dagger}$, J. Sokoloff ${ }^{\ddagger}$, S. Bolioli ${ }^{\S}$
}

\begin{abstract}
One solution to model quasi-optical systems - systems generally constituted by elements of large dimension with respect to the wavelength - is to use asymptotics, such as Gaussian Beams (GB) based techniques [1]. In these systems, dichroic surfaces are used to split, filter or mix an input signal. However, these surfaces are generally made of periodic elements of size that does not exceed a fraction of wavelength. Therefore, they cannot be modeled by such methods. In this paper we propose a technique, based on a spectral representation, to treat the interaction of a GB with a dichroic surface, we suppose that the fields reflected and transmitted by the interface can be approximated by GBs.
\end{abstract}

\section{Introduction}

In mm-wave radiometry, quasi-optical systems are used to guide, filter, split or mix the input signals. Such systems are generally constituted by the combination of different elements, e.g. mirrors, lenses, dichroic filters, or horn antennas. The number and the size of these elements render their modeling difficultly amenable by classical methods in acceptable computation times. For such systems, even physical optics may become computationally too costly. Gaussian beams constitute a possible alternative. The interaction of a GB with many of these elements is well-known (because their size is greater than few wavelengths). Indeed, in [2], Deschamps has demonstrated that the fields reflected and/or transmitted at a dielectric/metallic interface illuminated by an incident GB can be approximated by Gaussian beams. However, dichroic surfaces are in some cases structures which period may not exceed a fraction of wavelength. Thus they cannot be modeled by such methods.

One solution to integrate these elements consists in computing its response with the help of the method of moments coupled with Bloch/Floquet modal description. This allows the representation of the dichroic surface by reflexion and transmission coefficients.

In this article, we analyze the interaction between

\footnotetext{
${ }^{*}$ Service Antennes, CNES, 18 av. E. Belin, 31401 Toulouse, France, e-mail: kevin.elis@recherche.enac.fr

${ }^{\dagger}$ LETA, Département CNS, ENAC, 7 av. E. Belin, 31055 Toulouse, France

${ }^{\ddagger}$ LAPLACE, Université Paul Sabatier, 118 route de Narbonne, 31062 Toulouse, France.

$\S$ DEMR, ONERA, 2 av. E. Belin, 31055 Toulouse, France
}

a GB and a dichroic surface. We suppose that the fields reflected and transmitted by the interface can be approximated by GBs. The characteristics of these two GBs are found by means of a technique based on a spectral representation. Analytical expressions of the fields at any part of space are obtained via the asymptotic steepest descent path method.

This article is divided in five sections. The second section briefly presents GBs. The third section details the context and the configuration. The fourth section explains the principles of our method. The fifth section is dedicated to the results and comparisons with a reference method. In the last section, we conclude and discuss possible future works.

\section{Gaussian Beams}

\subsection{General points}

Gaussian beams are solution of the electromagnetic wave equation upon the paraxial approximation, which requires that the field is only weakly diverging with respect to its main propagation direction. The maximal angle of divergence generally admitted is of $20^{\circ}$. This study is in two dimensions with $\hat{\mathbf{y}}_{i}$ as the direction of invariance. The reference frame of the incident beam is $\left(0, \hat{\mathbf{x}}_{i}, \hat{\mathbf{z}}_{i}\right)$. In this article, we only present the case in which the electric field is tranversal to $\hat{\mathbf{z}}_{i}$ (TE). Note that the Tranversal Magnetic case (TM) can be formulated in a similar way.

\subsection{Used GBs}

In this article, we use a general formulation of GBs. This formulation is chosen to be as general as possible in order to be able to describe accurately the reflected and transmitted fields. A GB is characterized by a reference frame and four parameters : a complex amplitude $a_{i 0}$, a spatial shift $x_{i 0}$, a phase shift $\beta_{i}$ and a complex curvature radius $q_{i 0}$. We can develop $q_{i 0}$ as $j k \frac{W_{i 0}^{2}}{2}-z_{W_{i 0}}$, with $W_{i 0}$ the size of the beam waist, and $z_{W_{i 0}}$ the position of this waist along the propagation direction. In the plane $z_{i}=0$, the electric field is oriented along $\hat{\mathbf{y}}_{i}$ and given by

$$
u_{i}\left(x_{i}, 0\right)=a_{i 0} e^{j \frac{k}{2 q_{i 0}}\left(x_{i}-x_{i 0}\right)^{2}} e^{-j \beta_{i} x_{i}},
$$


We can deduct its plane-wave spectrum by means of a Fourier transform of (1). This yields

$$
\tilde{u}_{i}\left(k_{x i}\right)=a_{i 0} \sqrt{-j \frac{2 \pi}{k} q_{i 0}} e^{j \frac{\left(k_{x i}-\beta_{i}\right)^{2}}{2 k} q_{i 0}} e^{j k_{x i} x_{i 0}},
$$

with $k_{x i}$ the wave vector along the $x_{i}$ axis. This formulation is more general than those usually used. Indeed it includes several other formulations:

- For $z_{W_{i 0}}=0$, this formulation is identical to the Gabor phase-space beams [3] employed in the Gabor-Frame and Gabor-Base expansions.

- For $\beta_{i}=0$, this formulation is the same as the fundamental GB. Note that the 2D interaction of this type of GB with a dichroic surface as been studied in [5].

\subsection{Paraxial formulation}

In $\mathrm{TE}$, if the direction of propagation is along $\hat{\mathbf{z}}_{i}$, the paraxial vectorial expression is given by

$$
\begin{aligned}
& \mathbf{E}_{i}\left(x_{i}, z_{i}\right)=u_{i}\left(x_{i}, z_{i}\right) \hat{\mathbf{y}}_{i}, \\
& \mathbf{H}_{i}\left(x_{i}, z_{i}\right)=-\frac{1}{Z_{0}}\left(u_{i}\left(x_{i}, z_{i}\right) \hat{\mathbf{x}}_{i}-\frac{j}{k} \frac{\partial u_{i}\left(x_{i}, z_{i}\right)}{\partial x_{i}} \hat{\mathbf{z}}_{i}\right),
\end{aligned}
$$

where $\mathbf{E}_{i}$ and $\mathbf{H}_{i}$ are respectively the electric and magnetic fields, $Z_{0}$ the free space impedance, $k$ the wave number and $u_{i}\left(x_{i}, z_{i}\right)$ the paraxial formulation of the GB.

Assuming $\beta_{i}<k$ the GB is centered on a propagative plane wave. Its direction of propagation forms an angle $\alpha_{i}$ with the $z_{i}$ axis. This angle is given by

$$
\alpha_{i}=\arcsin \left(\frac{\beta_{i}}{k}\right) .
$$

We associate with this beam a new coordinate system

$$
\begin{aligned}
& x_{i}^{\alpha}=x_{i} \cos \left(\alpha_{i}\right)-z_{i} \sin \left(\alpha_{i}\right), \\
& z_{i}^{\alpha}=x_{i} \sin \left(\alpha_{i}\right)+z_{i} \cos \left(\alpha_{i}\right) .
\end{aligned}
$$

Via the steepest descent path method, we obtain the paraxial formulation of the beam

$$
u_{i}\left(x_{i}, z_{i}\right)=a_{i 0} \sqrt{\frac{q_{i 0}^{\alpha}}{q_{i}^{\alpha}\left(z_{i}^{\alpha}\right)}} e^{-j\left(\frac{k \alpha_{i}^{\alpha 2}}{2 q_{i}^{\alpha}\left(z_{i}^{\alpha}\right)}+\beta_{i} x_{i}^{\alpha}\right)} e^{-j k z_{i}^{\alpha}},
$$

with

$$
\begin{array}{r}
q_{i 0}^{\alpha}=q_{i 0} \cos ^{2}\left(\alpha_{i}\right), \\
q_{i}^{\alpha}\left(z_{i}^{\alpha}\right)=q_{i 0}^{\alpha}+z_{i}^{\alpha} .
\end{array}
$$

\subsection{Far-field formulation}

In the far-field zone, the paraxial approximation leads to an important phase error that can induce errors when suming beams with different directions of propagation. To overcome this problem, an alternative formulation exists that only depends on a far-field approximation. In spherical coordinates, this formulation is given by

$$
\begin{aligned}
& \mathbf{E}_{i}(r, \theta)=u_{i}(r, \theta) \cos (\theta) \hat{\mathbf{y}}_{i}, \\
& \mathbf{H}_{i}(r, \theta)=-\frac{u_{i}(r, \theta)}{Z_{0}}\left(\cos (\theta) \hat{\mathbf{x}}_{i}-\sin (\theta) \hat{\mathbf{z}}_{i}\right),
\end{aligned}
$$

with

$$
u_{i}(r, \theta)=a_{i 0} \sqrt{q_{i 0}^{\alpha}} \cos (\theta) e^{j \frac{k}{2}\left(\sin (\theta)-\frac{\beta_{i}}{k}\right)^{2} q_{i 0}^{\alpha}} \frac{e^{-j k r}}{\sqrt{r}} .
$$

\section{Context and configuration}

\subsection{Configuration}

We consider the configuration presented in Figure 1.

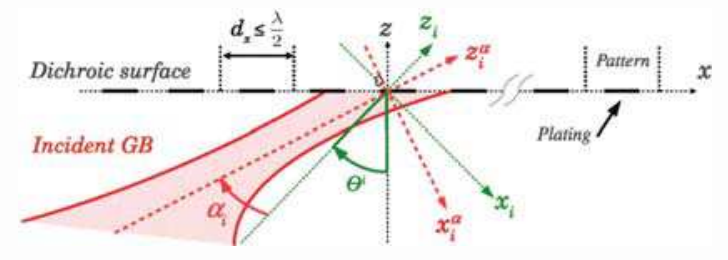

Figure 1: Configuration.

We suppose that the incident GB yields two beams. One transmitted and one reflected beam. Because the method is similar for both beams, only the study of the transmitted field will be presented in details.

\subsection{Dichroic surface}

The dichroic surface response, noted $\tilde{T}\left(k_{x}, z=0\right)$ is assumed to be already known, for example in a tabulated form. In practice, this response can be computed numerically using a method based on Floquet modes (for example via HFSS or [4]).

\section{Method}

The configuration is presented in Figure 2. The transmitted GB must be as close as possible to the transmitted field. Therefore, the goal is to determine its reference frame and four parameters. Its reference frame is chosen to be the same as for the incident beam.

To determine the four parameters, we make a matching of the angular spectrum of the incident and transmitted beams through the dichroic surface. In order to keep spectrum with Gaussian 


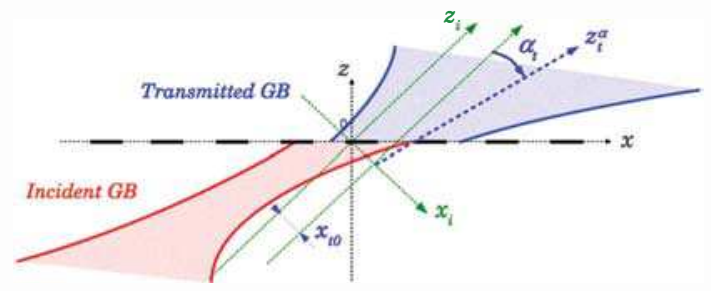

Figure 2: Configuration of the transmitted beam.

amplitudes, we choose to make this matching in the reference frame of the incident beam. In other words, the spectrum matching consists in writing

$$
\tilde{u}_{t}\left(k_{x i}\right)=\tilde{T}\left(k_{x i}\right) \tilde{u}_{i}\left(k_{x i}\right),
$$

with $\tilde{u}_{t}\left(k_{x i}\right)$, the spectrum of the transmitted field.

\subsection{Calculation steps}

\subsubsection{Transmitted beam}

In our method, there are three important steps. The first concerns the transmitted beam. We must express its angular spectrum in the reference frame of the incident GB. This spectrum is written as

$$
\tilde{u}_{t}\left(k_{x i}\right)=a_{t 0} \sqrt{-j \frac{2 \pi}{k} q_{t 0}} e^{j \frac{\left(k_{x i}-\beta_{t}\right)^{2}}{2 k} q_{t 0}} e^{j k_{x i} x_{t 0}}
$$

\subsubsection{Dichroic surface}

The second step concerns the dichroic surface. As for the precedent step, we must express its response in the reference frame of the incident GB. Neglecting evanescent waves, this amounts to

$$
k_{x i}=k_{x} \cos \left(\theta_{i}\right)-\sqrt{k^{2}-k_{x}^{2}} \sin \left(\theta_{i}\right) .
$$

The identification requires the following approximation of the transmission coefficient

$$
\tilde{T}\left(k_{x i}\right) \approx e^{T_{0}+T_{1} k_{x i}+T_{2} k_{x i}^{2}},
$$

where $T_{0}, T_{1}$ and $T_{2}$ can be complex. This approximation is obtained by means of a point matching technique on three points. One at the maximum of the incident beam and two at half of the maximum value.

\subsubsection{Identification}

The final step is the identification. From (2), (11) and (13) in (10), we obtain

$$
\begin{aligned}
& q_{t 0}=q_{i 0}-j 2 k T_{2}, \\
& \beta_{t}=\frac{\operatorname{Im}\left(q_{i 0}\right)}{\operatorname{Im}\left(q_{t 0}\right)} \beta_{i}+\frac{k}{\operatorname{Im}\left(q_{t 0}\right)} \operatorname{Re}\left(T_{1}\right), \\
& x_{t 0}=\frac{1}{k}\left(\operatorname{Re}\left(q_{t 0}\right) \beta_{t}-\operatorname{Re}\left(q_{i 0}\right) \beta_{i}\right)+\operatorname{Im}\left(T_{1}\right)+x_{i 0}, \\
& a_{t 0}=a_{i 0} \sqrt{\frac{q_{i 0}}{q_{t 0}}} e^{T_{0}+j \frac{1}{2 k}\left(q_{i 0} \beta_{i}^{2}-q_{t 0} \beta_{t}^{2}\right)} .
\end{aligned}
$$

Now we have all the parameters, we can used the equation (3) or (8) to compute the transmitted field.

\subsection{Simplified formulation}

When the response of the dichroic surface is slowly varying over $k_{x i}$, we can simplify the identification. Indeed, in this case $T_{1}$ and $T_{2}$ are equal to zero, which yields $q_{t 0}=q_{i 0}, \beta_{t}=\beta_{i}$ and $x_{t 0}=x_{i 0}$. This means that the transmitted GB is identical to the incident beam up to a coefficient. This coefficient is given by the response of the dichroic surface for $k_{x i}=0$.

\section{Simulation results}

\subsection{Dichroic surface without dielectric}

The first computed dichroic surface is infinitely plane and placed at $z=0$. The size of the period is $\lambda / 2$ with a perfectly conducting metallization of $0.1 \lambda$. The incident GB has a waist size of $\lambda$ positioned at $z_{i}=0$. Futhermore, $\beta_{i}=0$ and $\theta_{i}=45^{\circ}$. The frequency is of $200 \mathrm{GHz}$. The total near field is shown in Figure 3, on the left. The near field is compared to the one obtained with the reference method [4] on the right.

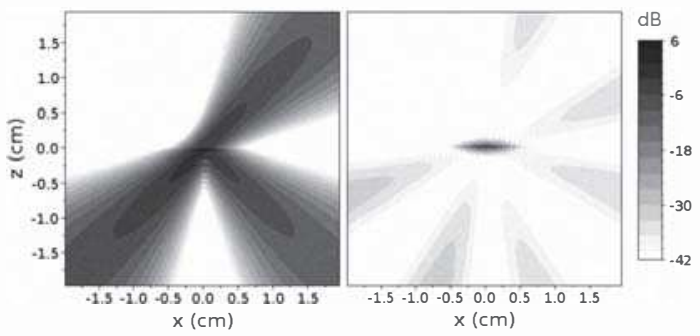

Figure 3: Total electric field (dB) with the spectrum matching (left), difference with MoM (right).

We can see in Figure 3 that our method gives very accurate results, with a difference below -40 
$\mathrm{dB}$. The only differences are due to neglecting the evanescent waves and assuming paraxiality of the incident beam. Indeed, we can see on the three GBs a residual error due to the near-field formulation, which is limited by the field paraxiality.

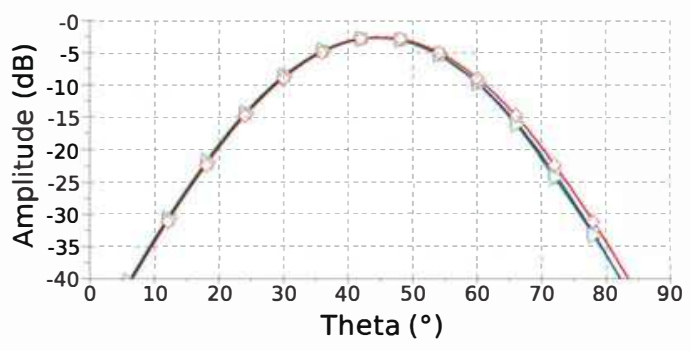

Figure 4: Normalized transmitted electric far-field $(\mathrm{dB})$ : MoM $(\triangleright)$, spectrum matching $(\square)$ and simplified formulation $(\diamond)$.

In Figure 4 we can see the far-field zone. The results are also very close to the reference method. This simulation leads to $\delta_{t} \approx 0.6^{\circ}$ and $x_{t} \approx 0.04 \lambda$. Note that to compute one hundred points, MoM needs $29.42 \mathrm{~s}$, spectrum matching only $33 \mathrm{~ms}$. We can see in Figure 4 that the field computed with the simplified formulation is less accurate but remain close to the reference solution.

\subsection{Dichroic surface on a dielectric}

This second dichroic surface is composed of elements of period $\lambda / 2$ with a perfectly conducting metallization of $0.1 \lambda$. It is laid on a dielectric having a thickness of $\lambda / 8$ and a relative permittivity of 2.1. We choose for the incident beam $W_{i 0}=2 \lambda$ and $z_{W_{i 0}} \approx 1.3 \lambda$. To test the method with a more demanding case, we take $\theta_{i}=70^{\circ}$. The frequency is the same as previously. The far-field is shown in Fig. 5.

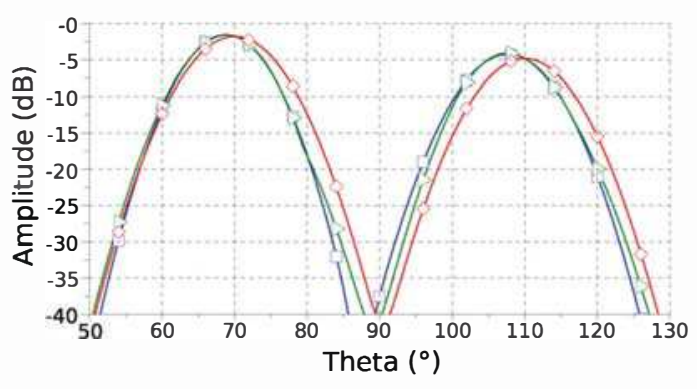

Figure 5: Normalized transmitted (left) and reflected (right) electric far-field : MoM ( $\triangleright)$, spectrum matching $(\square)$ and simplified formulation $(\diamond)$.
We observe in Figure 5 that the field is modified by the presence of the dichroic surface and applying a simple factor to the transmitted/reflected fields is not sufficient. We find $\beta_{t} \approx 0.024 k\left(\delta_{t} \approx 1.3^{\circ}\right)$ and $x_{t 0} \approx 0.7 \lambda$ for the transmitted field and $\beta_{r} \approx 0.04 k$ $\left(\delta_{r} \approx 2.3^{\circ}\right)$ and $x_{r 0} \approx 0.2 \lambda$ for the reflected field. Moreover, the complex curvature radii of the two FGs are significantely modified. In this case, the simplified formulation leads to an important error.

\subsection{Conclusion}

In this paper, we have analyzed the interaction of a GB with a dichroic surface. We have proposed a new method for approaching the transmitted and reflected fields by two GBs. This allows to obtain an analytical formulation of the field.

We have successfully tested this technique in two test cases. We have also noticed that in case where the dichroic surface only slightly modifies the incident field, transmitted and reflected GBs are identical to the incident beam up to a coefficient.

For future works, we intend to extend this theory to $3 \mathrm{D}$.

\section{Acknowledgment}

The authors would like to thank the CNES and the ENAC to fund this study.

\section{References}

[1] A. Chabory, J. Sokoloff, S. Bolioli, K. Elis, "Application of Gaussian-Beam based Techniques to the Quasi-Optical Systems of Radiofrequency Radiometers", EuCAP, Barcelona (Spain), april 2010.

[2] G.A. Deschamps, "Ray techniques in electromagnetics", Proceedings of the IEEE, vol. 60, No. 9, pp.1687-1693, february 1969.

[3] D. Lugara, C. Letrou, "Alternative to Gabor's representation of plane aperture radiation", Electronics Letters, vol. 34, No. 24, pp. 2286-2287, november 1998.

[4] R. Mittra, C. H. Cham, T. Cwik 'Techniques for analyzing frequency selective surfaces - A review", Proceedings of the IEEE, vol. 76, No. 12, pp. 1593-1615, december 1988.

[5] K. Elis, A. Chabory, J. Sokoloff, S. Bolioli "Intéraction 2D d'un faisceau gaussien avec une surface dichroïque pour la modélisation de systèmes quasi-optiques", JNM, Brest (France), may 2011. 